\title{
Application of MGS and ODY Aerobraking Accelerometer Data to Atmospheric Modeling
}

\author{
R. H. Tolson ${ }^{1}$ \\ North Carolina State University, Hampton VA 23666-6147 \\ G. M. Keating ${ }^{2}$ \\ The George Washington University, Newport News VA 23602
}

R. W. Zurek ${ }^{3}$

Jet Propulsion Laboratory, California Institute of Technology, Pasadena CA 91109-8099

S. W. Bougher ${ }^{4}$

University of Michigan, Ann Arbor, MI 48109-2143

C. J. Justus ${ }^{5}$

Morgan Research Corp. Huntsville, AL 35812

D. C. Fritts ${ }^{6}$

Colorado Research Associates Division, NorthWest Research Associates, Inc. Boulder, CO 80301

This paper reviews the use of accelerometer data for determining atmospheric density during the Mars Global Survey and Mars Odyssey missions and provides preliminary results from the Mars Reconnaissance Orbiter aerobraking operations. For MGS and ODY, accelerometer data were analyzed in both near real time and post flight to provide estimates of density, density scale height, latitudinal gradients, global longitudinal wave structure, and small scale (gravity) wave spectra. MGS (Odyssey) provided data during about 850 (300) passes at altitudes ranging from 100 to $160 \mathrm{~km}(95$ to 150$)$ and covering a latitude range of $60^{\circ} \mathrm{N}$ to $90^{\circ} \mathrm{S}$ (30N to 90N). A summary is given of the atmospheric phenomena encountered during the aerobraking phase of the missions and of some of the scientific results based on these data. MRO is expected to provide another $\mathbf{5 0 0}$ aerobraking passes over an altitude range from 95 to $170 \mathrm{~km}$ and from the south pole to the equator. These data provide powerful constraints on upper atmospheric models. Accelerometer and space craft requirements to enhance scientific return are discussed.

\footnotetext{
${ }^{1}$ Langley Professor, 100 Exploration Way, Hampton, VA 23666, Associate Member.

${ }^{2}$ Senior Research Staff Scientist, 1 Old Oyster Point Road, Suite 200, Newport News, VA 23602, Associate Member.

${ }^{3}$ MRO Project Scientist, MS 264-535

${ }^{4}$ Professor, Space Physics Research Laboratory, 2455 Hayward Avenue

${ }^{5}$ Senior Principal Scientist, EV13/Morgan, NASA Marshall Space Flight Center

${ }^{6} 3380$ Mitchell Lane, Boulder, CO 80301
} 


\section{Nomenclature}

$\begin{array}{llll}\mathrm{AB} & =\text { aerobraking } & \mathrm{h} & =\text { altitude, } \mathrm{km} \\ \mathrm{L}_{\mathrm{S}} & =\text { longitude of the mean sun along the martian equator } & \rho & =\text { density, } \mathrm{kg} / \mathrm{km}^{3} \\ \mathrm{LST} & =\text { local solar time, hrs } & \sigma & =\text { standard deviation } \\ \mathrm{MCO} & =\text { Mars Climate Orbiter } & & \\ \mathrm{MGS} & =\text { Mars Global Surveyor } & \\ \mathrm{MRO} & =\text { Mars Reconnaissance Orbiter } & \end{array}$

\section{Introduction}

Aerobraking is the utilization of atmospheric drag for beneficial orbit changes via multiple passes through an upper atmosphere. The first planetary application of aerobraking from which atmospheric properties were recovered was in 1993 during the Venus Magellan mission (Ref. 1) where aerobraking was performed for 70 days and about 750 orbital passes. Since accelerometer data were not available during that mission, atmospheric density (Ref. 2) at periapsis was recovered by the more traditional method of determination the change in the orbital period from tracking data. During Magellan Cycle 4, when periapsis was between 165 and $185 \mathrm{~km}$, spacecraft attitude data was used to estimate density and scale height (Ref. 3, Ref. 4) with very good agreement with the drag results. The second AB mission began in Sept. 1997 and ended with over 850 Mars Global Surveyor (MGS) AB passes (Ref. 5, Ref. 6). Mars Odyssey (ODY) followed in October 2001 with over 300 AB passes (Ref. 7). Mars Reconnaissance Orbiter (MRO) began AB in April 2006 and there are about 500 planned AB passes (Ref. 8, Ref. 9). All of the Mars spacecraft are equipped with three axis accelerometers with measurement noise below $0.3 \mathrm{~mm} / \mathrm{s}$ for a 1 second count and have allowed density recovery to better than $0.5 \mathrm{~kg} / \mathrm{km}^{3}$. For reference, nominal density during aerobraking is about $30 \mathrm{~kg} / \mathrm{km}^{3}$ and density scale height is around $7 \mathrm{~km}$. MGS (ODY) provided density data up to about 160 (150) $\mathrm{km}$. MRO accelerometer noise level is below $0.005 \mathrm{~mm} / \mathrm{s}^{2}$ equivalent to a density of about $0.01 \mathrm{~kg} / \mathrm{km}^{3}$.

As seen in Fig. 1, these missions also cover a significant fraction of the seasonal (Ls), diurnal (LST) and latitudinal space of interest for validation of atmospheric models. In the three dimensional cube, these mission curves leave a lot of voids, but in the two dimensional projections, only the southern hemisphere spring-summer quadrant remains unsampled. The opportunity exist to sample this region with a 2011-2012 aerobraking mission.

This paper will review some of the atmospheric density results, will present some new measurements that might be extracted from the data, and discuss potential benefits of more precise accelerometer measurements and some of the limiting factors.

\section{First Impressions}

It had been suggested before the MGS mission that the Martian thermosphere demonstrated between 30\% to $40 \%$ short time scale variability and that short wave length gravity waves might propagate to aerobraking altitudes. Numerous AB passes
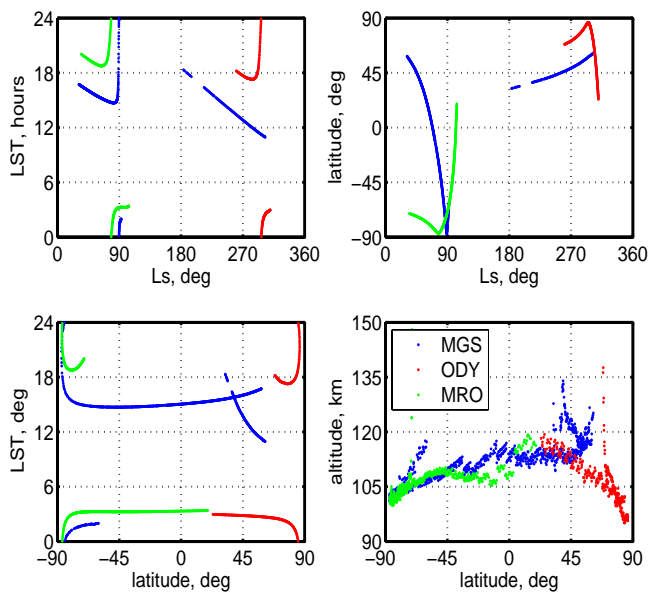

Figure 1. Span of atmospheric parameters covered by MGS, Odyssey and MRO aerobraking. confirmed those suggestions. Fig. 2 provides evidence of significant wave structure during four typical ODY AB passes. The blue lines are the recovered density from the accelerometer data and the red lines are MarsGRAM (Ref. 10, Ref. 11) profiles that have been scaled to provide the same drag effect or approximately the same area under the profiles. During Odyssey this scale factor was determined for each orbit and varied from 0.35 to 0.8 (Ref. 12). Through MRO orbit 225 the scale factor has varied from 1.8 to 5.5. ODY orbits 155 and 157 were during a phase of the mission where AB was in or near the polar vortex. The outbound leg of orbit 155 shows the traditional bell shape and closely follows the scaled MarsGRAM profile. From zero to $200 \mathrm{sec}$. the vehicle latitude is north of $82^{\circ} \mathrm{N}$ and the variability is typical of the low small-scale variability observed while inside the vortex. The "bump" in the inbound leg at $-100 \mathrm{sec}$ is about twice the equivalent outbound 
density and occurs at $75^{\circ}$ latitude. Periapsis occurs at about $82^{\circ} \mathrm{N}$ latitude and $270^{\circ} \mathrm{E}$ longitude. Orbit 157 covers the same latitudes as orbit 155 but with periapsis at $162^{\circ} \mathrm{E}$ longitude. The respective periapsis altitudes are 101.2 and $102.3 \mathrm{~km}$ and the latitudes and LST are essentially the same. Derivation of a density scale height from accelerometer data is complicated by the combined response to both horizontal and vertical density gradients projected along the spacecraft trajectory during an aeropass. The density scale height at periapsis is about $10 \mathrm{~km}$. Based on altitude difference only, a $11 \%$ change in periapsis density would have been expected. Factors of two change in maximum density from orbit to orbit can, and do, occur, as shown here. On both orbits a maximum latitude of $87^{\circ}$ was reached about 100 sec. after periapsis.

The density scale height of $10 \mathrm{~km}$ in the lower thermosphere implies an atmospheric temperature between 140 and $150 \mathrm{~K}$, which is much warmer than expected or experienced on MGS. Post flight analysis showed a strong latitudinal temperature gradient increasing toward the pole, suggesting a polar warming. Ref. 20 interprets this warming as due to strong southern hemisphere summer solar and dust heating near perihelion. This heating induces strong interhemispheric circulation and increased adiabatic heating near the north pole.

By P199, periapsis had precessed farther south to $72^{\circ} \mathrm{N}$ and the latitude is north of $78^{\circ}$ from 75 to 400 sec. The inbound leg had 5 waves above periapsis. For these waves, also shown vs. altitude in Fig. 3, the peak to trough density ratios vary between 1.2 and 1.9 and the peaks are about $2^{\circ}$ apart in latitude and increase from 1 to $3 \mathrm{~km}$ in vertical wavelength. The last peak just after periapsis is followed by a low variability outbound leg after 75 sec. at a latitude of $78^{\circ}$. The interpretation is that the vortex boundary is near $76^{\circ}$ giving a highly variable profile up to $75 \mathrm{sec}$. while outside the vortex and low variability inside the vortex after $75 \mathrm{sec}$. Just before periapsis there is more than a factor of 2 decrease in density in about 10 seconds as the altitude decreased by less than $1 \mathrm{~km}$. Such rapid decreases are not uncommon and occasionally occur over shorter spatial scales.

In Fig. 3 limited wave structure appears from $115 \mathrm{~km}$ to $130 \mathrm{~km}$ altitude and may then start again above $130 \mathrm{~km}$. Somewhat similar structure above $130 \mathrm{~km}$, may suggest the upper atmosphere waves have a large latitudinal span.

Finally, nearly circular orbit 280 is included to show that local phenomena can produce nearly factor of 2 changes in density over very short time scales. The latitude range is from $35^{\circ} \mathrm{N}$ at $-200 \mathrm{sec}$ to $57^{\circ} \mathrm{N}$ at $+200 \mathrm{sec}$. There is considerable variability from a mean profile on both the inbound and outbound legs. The spike just after periapsis has a latitude width of about $2^{\circ}$. It should be kept in mind that, for the $\mathrm{AB}$ missions, the solar array temperature is the limiting factor and that thermal capacity and conduction through the solar array smooths many of these short term variations and such local peaks may thus contribute little to the solar array maximum temperature.
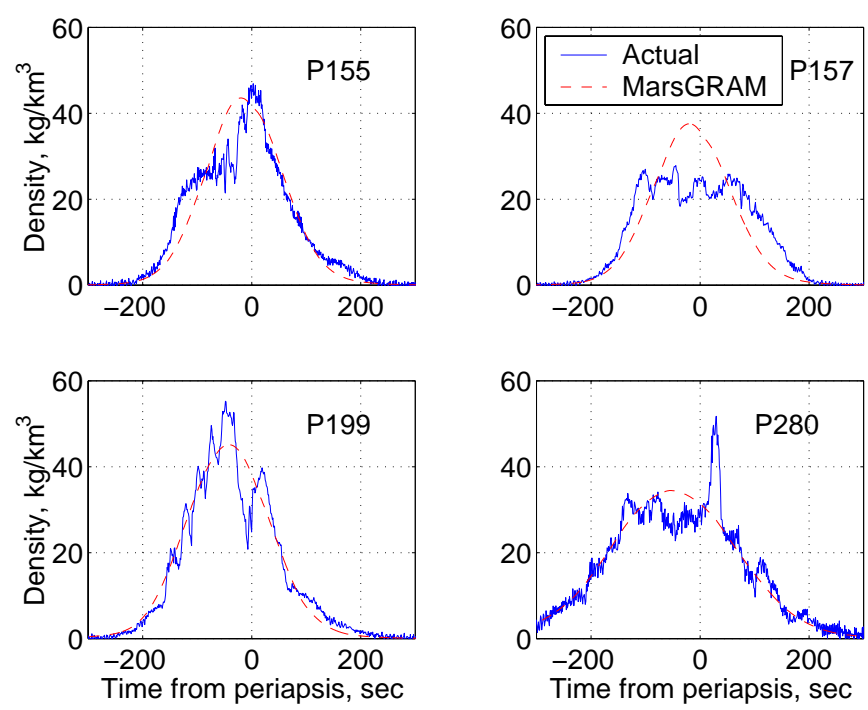

Figure 2. Four typical Odyssey density histories.

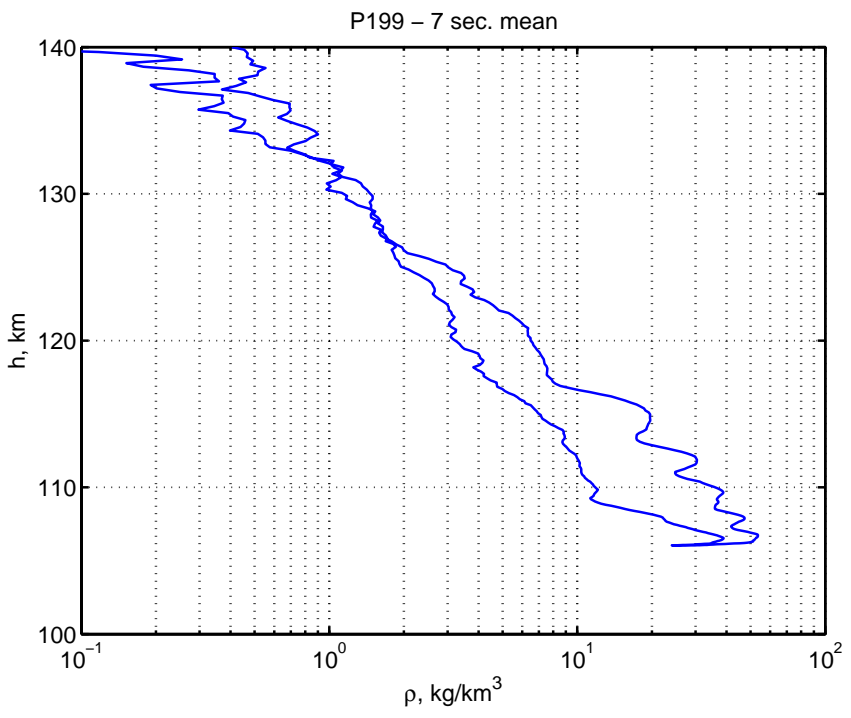

Figure 3. P199 density profile after 7 second averaging. Altitude above reference ellipsoid.

3

American Institute of Aeronautics and Astronautics 


\section{Gravity Waves}

The short wave length variations along an orbital pass have been generally attributed to gravity waves. Studies are underway to characterize the altitudinal, latitudinal and seasonal variability and to correlate these with topography and the mean jet. A first cut at the variability during each orbit is illustrated for orbit 199 in Fig. 4. The short wave length waves are extracted by comparison of the 1 sec. data with the 39 second running mean. The $\mathrm{s} / \mathrm{c}$ had a horizontal velocity of about $4 \mathrm{~km} / \mathrm{s}$ so the averaging is over about $1600 \mathrm{~km}$ along track. From Fig. 3, the altitude range covered in Fig. 4 is from 105 to about $120 \mathrm{~km}$. The lower chart shows horizontal wavelengths of about $100 \mathrm{~km}$. Separating the vertical and horizontal structure is part of an ongoing analysis. Nevertheless, this ratio of densities is a metric for gravity wave activity and for orbit 199 had a standard deviation of 0.12 .

This type of analysis was performed on each orbit for both MGS and Odyssey resulting in Fig. 5. The red dots are for orbits $40\left(35^{\circ} \mathrm{N}\right)$ up to $201\left(60^{\circ} \mathrm{N}\right)$ during winter and including the Noachis storm (Ref. 13). Aerobraking begins again in the spring (blue) on orbit 587 at $60^{\circ} \mathrm{N}$ and $\mathrm{L}_{\mathrm{s}} \sim 30^{\circ}$ (Fig. 1) with considerable less variability. As periapsis precesses across the equator toward $60^{\circ} \mathrm{S}$ the variability increased, perhaps due to the presents of the winter jet permitting high altitude gravity wave propagation. After periapsis passed over the pole (magenta) and into the night, the same variability was observed back to $60^{\circ} \mathrm{S}$. There is
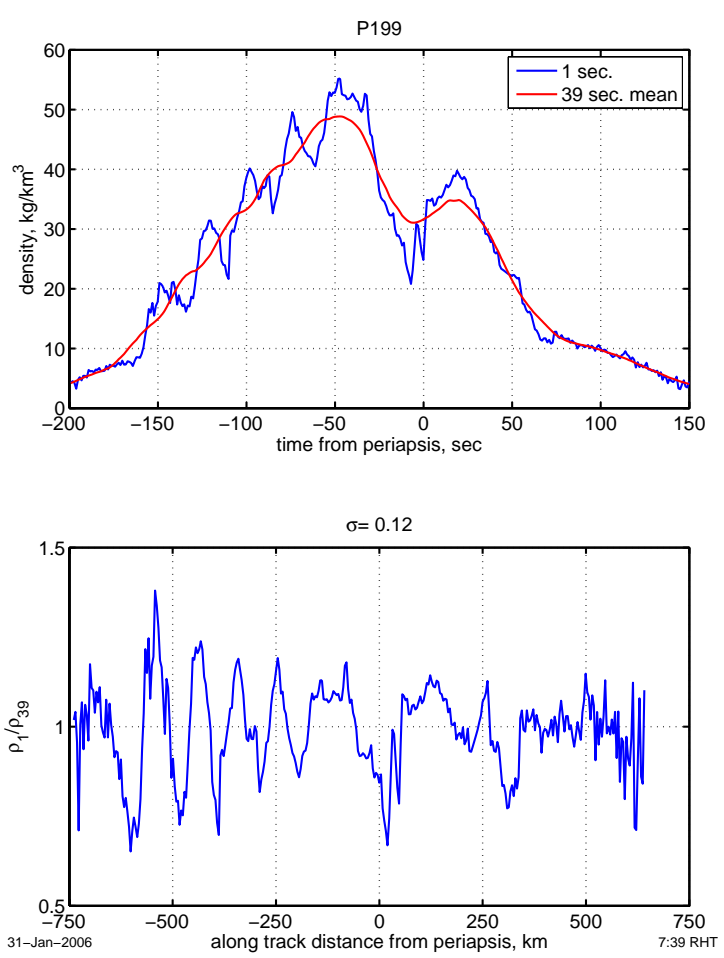

Figure 4. P199 density history and ratio of $1 \mathrm{sec}$. samples to 39 second running mean.

some similarity between the latitudinal variation near $60^{\circ}$ in both winter hemispheres, suggesting a similar underlying cause. As of this writing, MRO has been AB poleward of $70^{\circ} \mathrm{S}$ and the corresponding sigma has been less than 0.08 . This is up to twice the MGS values at about the same latitude, LST and $\mathrm{L}_{\mathrm{S}}$ (Fig. 1).

As ODY periapsis approached the winter pole (lower panel, blue), variability showed a signal similar to the south pole results for MGS, but the magnitudes were significantly larger. The results for ODY south of $45^{\circ} \mathrm{N}$ should be ignored, per later discussion on data noise.

Though limited in latitudinal and seasonal range, studies (Fritts, 2006) of the ODY variances, like those shown in Fig. 4, led to a number of results. For orbits 7 to 134 , it was found that there is a localization of high variance in the $130^{\circ}$ to $180^{\circ}$ longitude and $120-140 \mathrm{~km}$ altitude ranges and that the altitudinal growth appears to be slower than expected for non-dissipating waves. Due to the limited sampling, it is difficult to separate spatial and temporal variations. Nevertheless, high spatial and temporal variability suggest filtering by large-scale waves or tides. Wave spectra exhibited consistent shape with altitude, but amplitude growth was much smaller than expected in the absence of dissipative processes. Gravity wave momentum fluxes estimates were much
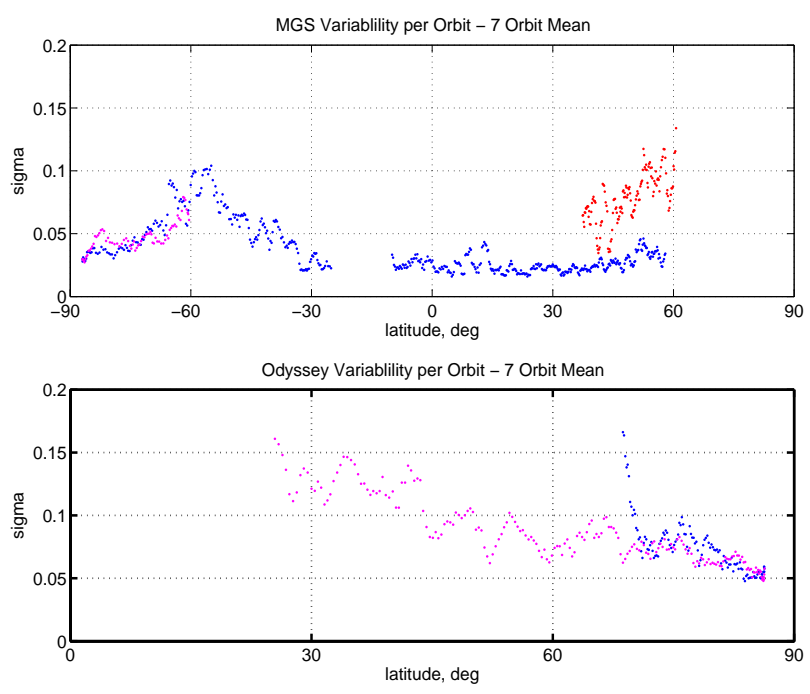

Figure 5. MGS and ODY short wavelength variability during each orbit, 7 orbit average. 
larger than comparable Earth fluxes, adding additional evidence that gravity waves have significant influence on large scale motion.

\section{Orbit to Orbit Variability}

One of the statistics of interest to $\mathrm{AB}$ operations is the orbit to orbit variability. This parameter, called persistence, is calculated using the density profile from one pass to predict the density at the periapsis altitude of the next pass. Since altitude, latitude and LST change so little from pass to pass, an exponential density variation with altitude is fit by least squares to determine density at periapsis and the local scale height, which is typically between 6 and $8 \mathrm{~km}$. This simple model is used to predict the density at the next periapsis altitude. If no maneuvers are used to raise or lower periapsis, subsequent periapsis, perturbed primarily by the non-central gravity field, are generally within 1.5 $\mathrm{km}$ altitude of each other.

Fig. 6 shows the ratio of the density recovered from the MGS accelerometer data to the predicted density from the previous orbit. It is seen that persistence overestimates by about $6 \%$ and that the average orbit to orbit variability is consistent with the preflight estimate of $40 \%, 1 \sigma$, but there is significant latitudinal-seasonal structure in the variability.

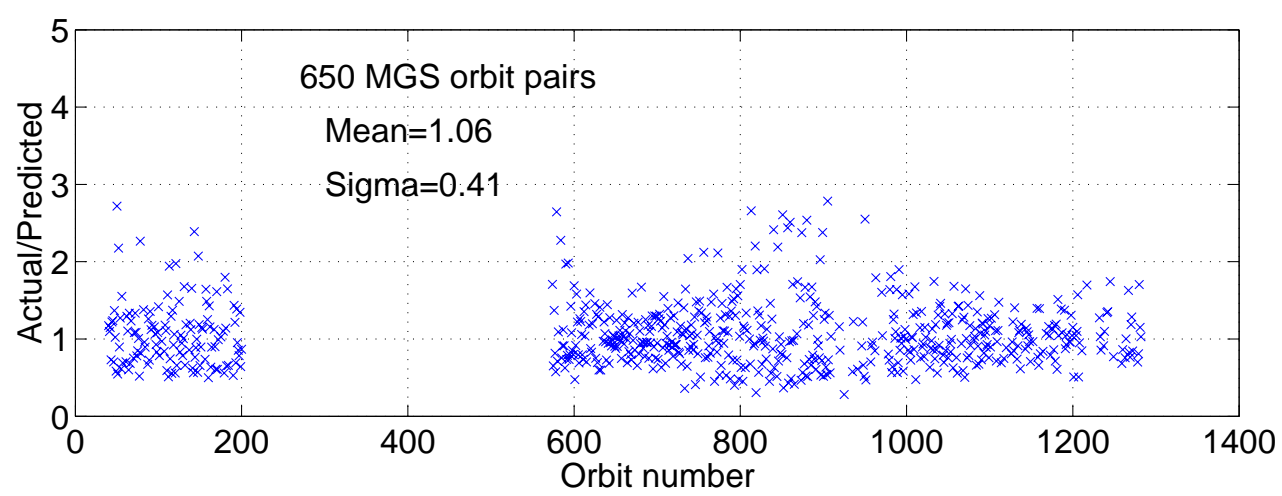

Figure 6. MGS orbit to orbit persistence.

For the first 200 orbits in Fig. 6, the MGS periapsis precesses from about $35^{\circ} \mathrm{N}$ to $60^{\circ} \mathrm{N}$ during northern winter. The Noachis dust storm (Ref. 13) occurred near orbit 50 causing more than a 100\% increase in density. The increase in variability as periapsis approaches the pole could be due to the dust storm produced general atmospheric variations or due to periapsis approaching the polar vortex. After the hiatus, phase 2 began with high variability at a latitude of $60^{\circ} \mathrm{N}$, decreases significantly at $30^{\circ} \mathrm{N}(\mathrm{P} 700)$ and is a maximum near the equator (P850). Global scale stationary waves were present throughout this region (Ref. 13). Periapsis reaches the south pole on orbit 1188 and Ls $=90^{\circ}$ and the reduced variability occurs most likely due to being inside the polar vortex, although this has not been verified by reanalysis of the data.

Fig. 7 shows an example of the "stationary" waves encountered during MGS AB. Densities were determined at reference altitudes at $10 \mathrm{~km}$ intervals. The "+" in the plots represents these data. The blue line is a stationary wave 4 fit to the data where the waves are assumed to be stationary in longitude ("s-wave" number will be used to denote the stationary waves observed during $A B$ to distinguish these waves from the conventional atmospheric wave number). The red lines are the $1 \sigma$ deviation based on the formal statistics. At the lower altitude, nearest to where AB occurs, the maximum to minimum density ratio is about 3. During MGS operations, these types of model were used to improve prediction capability. It is seen that these s-waves maintain phase across the entire range of altitudes at which density can be extracted from the data. Shortly after the end of MGS AB, a model was developed for use in MCO AB design (Ref. 14). The model provides the amplitude and phase of s-wave 1 through 3 along the nearly constant density surface represented by MGS AB altitude. Latitudinal amplitude variations in both s-wave 1 and 2 strongly suggest a cellular structure. A significant increase in amplitude of s-wave 1 was also noted during the Noachis dust storm without a change in longitudinal phase. Longitude phase shifts due to either latitudinal or seasonal changes occurred rapidly for s-wave 2 and much more slowly for s-wave 1 . 
Although not recognized during MGS operations, the s-wave 2 component was anticipated pre-flight (Ref. 15) as the result of the westward propagating wave 1 diurnal Kelvin wave. Because this Kelvin wave is in near resonance with the rotation of Mars, the wave appears nearly stationary to a sun synchronous satellite. Due to the satellite being in a near sun synchronous orbit, other waves have also been attributed to lower atmosphere non-migrating tides that appear to be stationary (Ref. 16, Ref. 17). The s-wave 3 component is "largely due to a wave 2 Kelvin mode and an eastward-propagating wave 1 semi-diurnal tide” (Ref. 17). A comparison of GCM derived density wave component amplitudes shows (Ref. 16) very good agreement with accelerometer derived densities s-wave amplitudes. ODY did not show the same stationary wave features as were found with

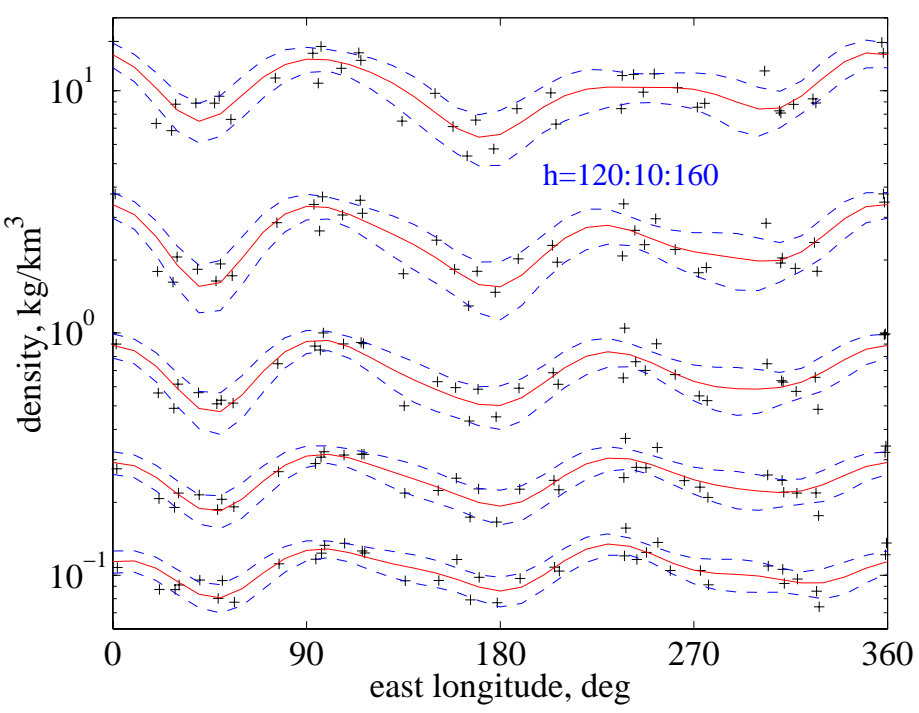

Figure 7. "Stationary” waves derived from orbits 587-626. MGS, perhaps because of the limited latitude coverage.

Similar to Fig. 6 for MGS, Fig. 8 shows the ratio of the density recovered from the Odyssey accelerometer data to the predicted density from the previous orbit. It is seen that persistence overestimated by $10 \%$ and that the average orbit to orbit variability is nearly $50 \%, 1 \sigma$. From orbit 20 to 75 , the latitude of periapsis was increasing from $70^{\circ}$ to $80^{\circ} \mathrm{N}$ and the lack of persistence was attributed to crossing the horizontal density gradients associated with the polar vortex. However, after precessing over the pole, the same latitude band was crossed during orbits 160 to 200 without a significant increase in orbit to orbit variability. Local solar time changed from late evening to early morning between these intervals (Fig. 1) but $\mathrm{L}_{\mathrm{S}}$ did not change significantly. The high variability after orbit 250 is an artifact due to (1) a doubling of the accelerometer noise at P275 and (2) increasing periapsis altitude during the "walkout" phase with resulting reduction in accelerometer signal to noise ratio.

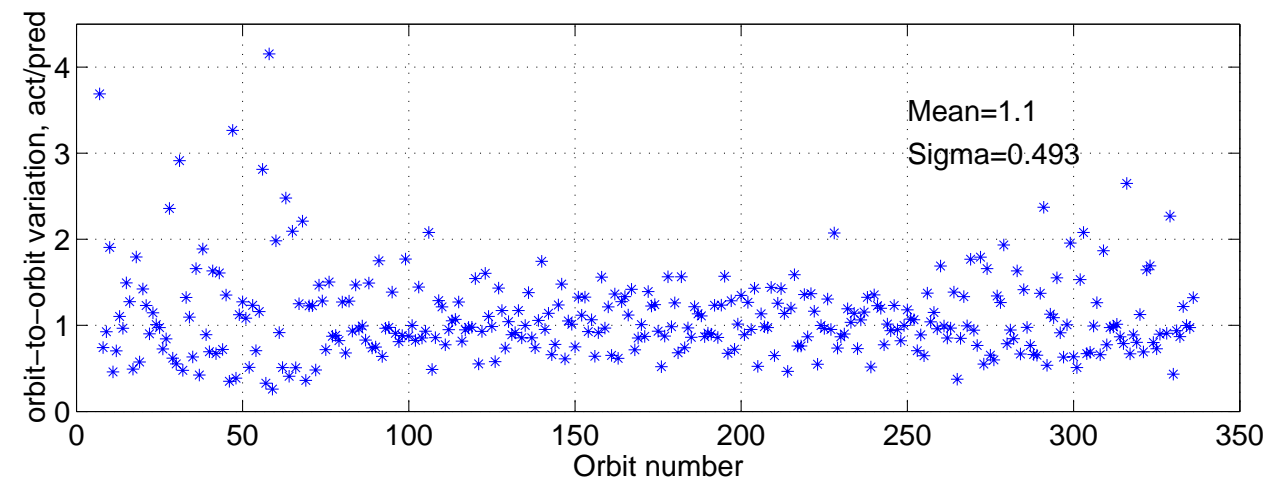

Figure 8. Odyssey orbit to orbit persistence.

\section{Cross Wind Recovery}

The inherent aerodynamic stability of AB vehicles provides the opportunity to recover winds that influence either the pitch (vertical winds) or yaw (cross winds) of the vehicle. Along track winds are probably too small, relative to the vehicle velocity of $4 \mathrm{~km} / \mathrm{s}$, to be recovered. Since both MGS and ODY performed AB with the solar arrays nearly 
horizontal, the vehicle orientation is most sensitive to a cross wind. Both orbits have $93^{\circ}$ inclinations thus, except near the pole, so this component is essentially the zonal wind throughout most of the orbit. During an AB pass, both MGS and ODY weather vane into the apparent wind. Deviations from the trim orientation produce aerodynamic torques on the body which can be calculated from attitude rate information (Ref. 18). The yaw angle to the relative wind can then be recovered from the aerodynamic moment data base. Finally, the cross wind is determined from the angle between the inertial velocity and relative wind direction.

Accelerometer data provide an independent solution by using the ratio of the horizontal acceleration to the axial acceleration. Again from the aerodynamic force data base the yaw angle to the relative wind is determined. The vehicle attitude is known from the onboard guidance system, so the yaw angle can be determined and thence the cross wind. During a typical AB pass with a peak density of $30 \mathrm{~kg} / \mathrm{km}^{3}$ the drag acceleration reaches about $15 \mathrm{~mm} / \mathrm{s}^{2}$. The ODY (MRO) noise level of $0.08(0.005) \mathrm{mm} / \mathrm{s}$ is equivalent to a change in heading of about $0.005(0.0003)$ radians. This maps into a cross or vertical wind sensitivity of $20(1.5) \mathrm{m} / \mathrm{s}$. Clearly there is significant potential in directly recovering winds from accelerometer data.

Unfortunately, these processes have random noise components and biases. One of the potential biases is due to errors in the location of the center of mass of the vehicle. Center of mass location errors directly bias the aerodynamic moment coefficients. Both methods depend on the aerodynamic data base and although the data base may be adequate for mission design and operation, there is no guarantee it is adequate for wind recovery. Further, the two approaches are not completely independent in free molecular flow since the dominate force is drag and moments and forces are linearly dependent. Few orbits get into the strong transitional flow regime where the methods would not be related.

Nevertheless, aerodynamic torque data have been used to obtain a mean cross wind during MGS AB (Ref. 18) and the results compare favorable with upper atmospheric GCM predictions even though there are potential biases due to the broken solar array. Odyssey provides a much more favorable opportunity to recover winds and studies have been proposed to do wind comparisons with winds from thermospheric models (Ref. 19). A preliminary result is shown in Fig. 9. The upper panel provides the recovered wind. The blue line is derived from the aerodynamic force database and the green line from the moment data base. The overall trend in the winds is generally the same for either recovery. However, the vertical location of the moment derived winds is very sensitive to the location of the center of mass. Center of mass (CM) varies throughout the mission due to the use of attitude control fuel. Another example of engineering data required to recover atmospheric properties during $\mathrm{AB}$. The results in the figure were derived by solving for a fixed CM over a 20 orbit interval. The vehicle passes closest to the pole at about 40 seconds after periapsis and the winds are nearly zero as would be expected if the winds were purely zonal. This is not a consistent result through the full orbit set. For purely zonal winds, the cross wind changes direction in the spacecraft frame upon pole crossing as shown in the figure. The winds are clearly stronger on the inbound leg than on the outbound suggesting that the polar vortex is not symmetrical.
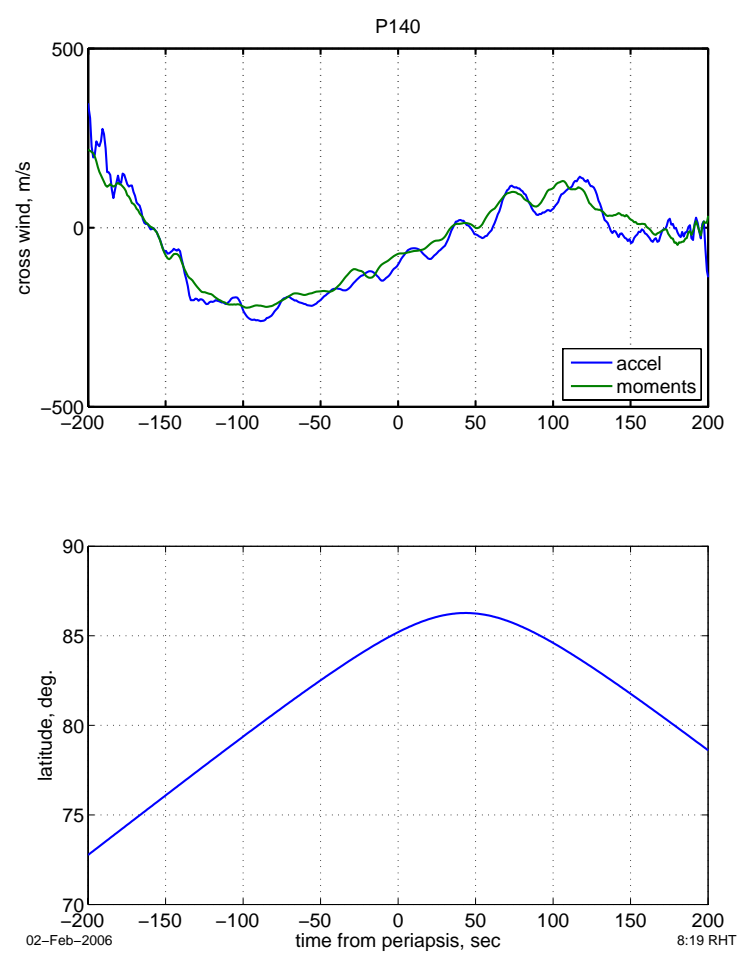

Figure 9. Inferred cross winds for ODY orbit 140. 
Additional evidence of the polar vortex and the asymmetry is shown in Fig. 10. In an attempt to understand the temporal and spatial variations, polar plots of the density variation were made daily for orbits from the previous days. One such polar plot is shown in Fig. 10. Each track on the plot shows the density along the orbit path on a latitude-longitude polar plot. All of the measured densities have been mapped to a common reference altitude, in this case $100 \mathrm{~km}$. Periapsis is identified by a star on each track. The time between close ground tracks (e.g. 160 and 167) is about 1 sol and such adjacent pairs were compared to generate confidence in the mapping and to identify trends in the variations in the density field. A strong wave 1 pattern is clearly evident at this time, with maximum density occurring in the first and fourth quadrants. Further, along track variability is small within $10^{\circ}$ of the pole and increases as latitude moves south. Along track variability is most apparent in orbits 159, 165, and 166. The strong latitudinal density gradient away from the polar region is also quite evident, particularly in the first and fourth quadrants.

\section{Characterizing the Accelerometer Data}

The basic output of the accelerometers is the change in velocity over the sample time, so noise is quoted in change in velocity instead of acceleration. Depending on the implementation, sample rate may influence the conversion to acceleration accuracy. Throughout of the MGS mission the data noise and the bias were essentially constant (Ref. 5). The data noise was constant because every sample was transmitted. Bias was nearly constant because the accelerometer block had an active temperature control system. Odyssey, on the other hand, showed bias shifts at about 1/5 of the noise level across an AB pass (Ref. 7). Odyssey accelerometer bias often increased rapidly at the end of the pass. The cause was likely aerodynamic heating of the bus, however there were no IMU temperature measurements available. Further, there was some sort of double digitization inside the IMU which resulted in nonstationary noise. Due to onboard memory limitation, the sample size was reduce twice during the mission and due of the sampling process the coherence between the data was lost.

Fig. 11 shows the noise level derived from data collected outside the atmosphere. For the first 138 orbits, the noise level of $0.086 \mathrm{~mm} / \mathrm{s}$ is fairly close to the least significant bit value of $0.0758 \mathrm{~mm} / \mathrm{s}$. The influence of changing the number of high rate samples from 200, to 50 and then to 20 is clearly evident. The lowest noise level corresponds to determining density to about $0.15 \mathrm{~kg} / \mathrm{km}^{3}$ and the largest to about $1.1 \mathrm{~kg} / \mathrm{km}^{3}$. This increase in noise level reduced the applicable altitude range of the data by 2 scale heights or between 15 to $20 \mathrm{~km}$. MRO accelerometers are a modification of the models on ODY and have a noise level of between 2 and 5e-3 $\mathrm{mm} / \mathrm{s}^{2}$ or about 30 times smaller than the best ODY data. Based on noise level only, the sensitivity may be

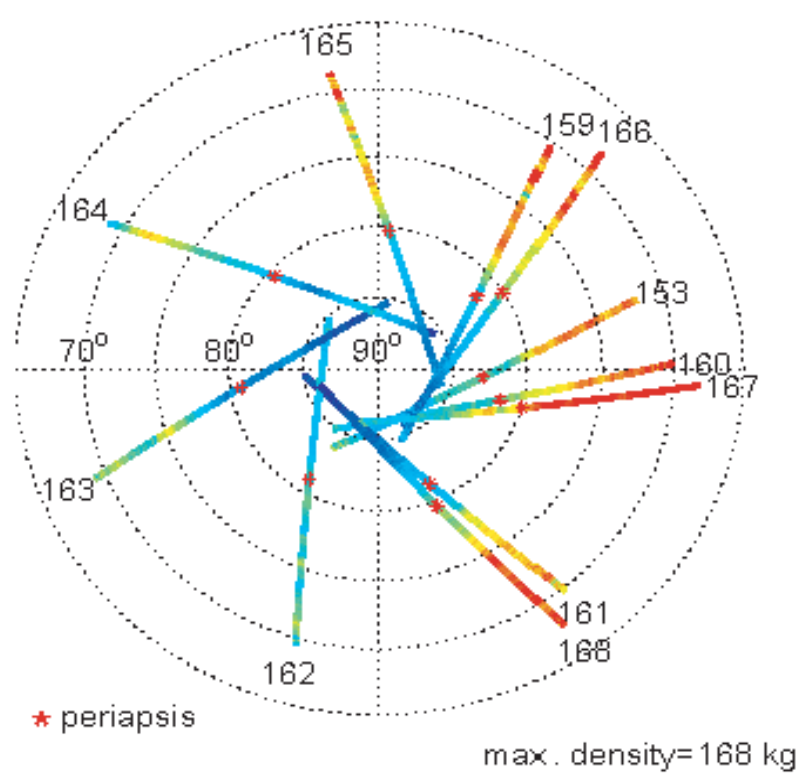

Figure 10. Polar plot of densities mapped to 100 km altitude for orbits 153 and 159 through 168. Color scale from 20 to $100 \mathrm{~kg} / \mathrm{km}^{3}$ and density truncated at $100 \mathrm{~kg} / \mathrm{km}^{3}$ to enhance variability.
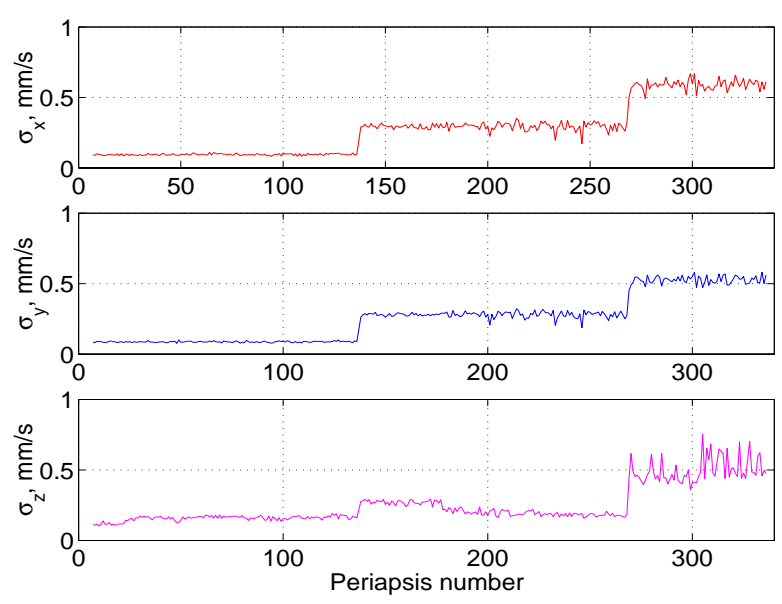

Figure 11. Variation in accelerometer noise level throughout the ODY mission. 
sufficiently to measure density up to nearly $200 \mathrm{~km}$. However, as of this writing, there are unresolved issues that might degrade the minimum density recoverable.

\section{Other Data Types}

The accelerometers are seldom mounted sufficiently close to the center of mass to ignore the accelerations due to the angular motion of the vehicle. During AB, the attitude control system is generally in the reaction control mode with a wide angular dead band and rate limited. Consequently the body rates can become significant contributors to the measured acceleration. Off-the-shelf rate gyros generally have sufficient accuracy to correct the accelerometer measurements to the noise level, assuming the relative location of the accelerometers to the center of mass is accurately estimated pre-flight or calibrated in flight.

A typical history of the body rates for ODY are shown in Fig. 12. The $\mathrm{x}(\mathrm{z})$ rates are shifted up (down) for clarity. The $y$-axis is into the wind and had no aerodynamic restoring force. The other two axes are stable, with the $\mathrm{x}$-axis being the most stable. Motion about all three axes are coupled through the cross products of inertia and on board momentum supplied by the reaction wheels. The angular acceleration derived from these rates was the major contributor to the correction of the measured acceleration. For typical AB passes, the maximum contribution is less than $0.5 \mathrm{~mm} /$ $\mathrm{s}^{2}$, which is larger than the noise level. From the y-axis angular rate data it is seen that large thruster firings took place at -160 and -75 seconds. Though more difficult to see, there are nearly continuous small firings from -75 seconds through about 120 seconds. These particular attitude control jets are coupled and theoretically

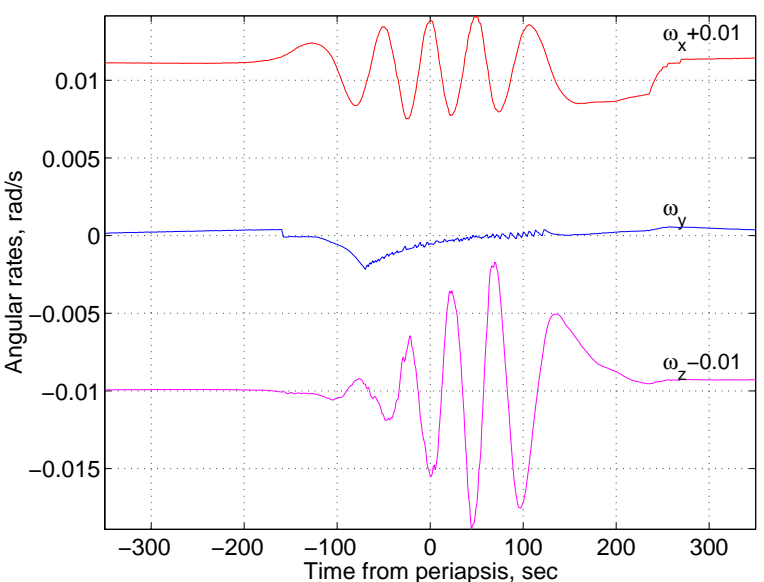

Figure 12. Body angular rates during ODY P076, rates displaced for clarity produce no net acceleration of the s/c. However, residual imbalances remain and short impulses are very difficult to calibrate due to the sensitivity of the total impulse to initial catalyst temperatures and other parameters. For MRO the minimum attitude thruster firing introduces a change in velocity about 3 times the noise level on the accelerometer data. MRO telemetry contains temperature data for each accelerometer and the thruster catalyst bed temperature. Post flight analysis will determine the value of these data in improving accelerometer data quality.

\section{Lessons Learned}

A well designed experiment to recover atmospheric density requires numerous supplemental data sets. Further, a systems approach to the overall design of the vehicle is required to extract the most information from the data. For example, potential degradation of the accelerometer data can result from structural vibration or fuel slosh. The broken solar array on MGS introduced a 6-7 Hz oscillation and the archived data set is a 7 point mean of the 1 second density recoveries. This vibration degraded the potential horizontal spatial resolution from 4 to $25 \mathrm{~km}$. Generally structural vibration is sufficiently small and of high enough frequency to have a ineligible influence on the density recovery. Fuel slosh is a different issue. While out of the atmosphere, the fuel distribution in the tanks is controlled by surface tension. As the vehicle enters the atmosphere, drag eventually overcomes surface tension and the fuel rushes forward. The center of mass of the vehicle moves relative to the accelerometers and produces an artifact in the data. It is though that any oscillation is rapidly damped because drag is rapidly increasing on the inbound leg. On exiting the atmosphere the process is reversed, but now the fuel can slosh around under the weak surface tension forces. Slosh oscillation periods are difficult to estimate and can be of the order of 10 to 100 seconds and could introduce artifacts that might be misinterpreted as density waves in the upper atmosphere.

Geometric symmetry and simplicity are also highly desirable for density or wind recovery. The fidelity of the aerodynamic data base is likely diminished for asymmetric bodies or bodies that have complicated geometries. During the inbound leg, the dynamic pressure increases and attitude oscillations are damped. After maximum dynamic pressure is reached, the attitude oscillations rapidly increase and the vehicles often experiences large yaw or pitch angles. Vehicles with complicated geometries make accurate aerodynamic calculation more difficult at these 
high angles. For a dedicated aeronomy mission that depends on accelerometer data, a system study should be performed to trade tighter attitude rate control, thruster torque unbalance, attitude fuel usage, and aero data base accuracy to optimize the science return.

\section{Conclusions}

MGS was the first Mars mission where an accelerometer experiment was project approved. The experiment was not an official science experiment and had none of the privileges given to such experiments. When aerobraking began, of the 10 samples per second collected on board the vehicle, two samples were transmitted each second and all 10 were sent every 8 seconds. There was not enough telemetry bandwidth to include more accelerometer data because of all the other engineering data required to monitor the health of the spacecraft. The two sample per second data proved useless, but the full sample every 8 seconds provided reasonable profiles and showing curious features. On orbit 15 the solar array deflected 10 degrees from its nominal position and the project realized it had a major anomaly. After aerobraking began 20 orbits later, all ten samples were sent every second because one way of monitoring the vibration of the solar array was with the accelerometer data. This serendipitous data set led to discoveries of the planetary scale waves, the gravity waves, and the incredible latitudinal, seasonal and diurnal variability of the atmosphere. These data still provide the basis for numerous ongoing scientific studies. So there was at least one good outcome from the broken solar array.

The great variability of the atmosphere, discovered by MGS, added a level of risk to future AB mission. Consequently, real time analysis of accelerometer measurements during $\mathrm{AB}$ operations became a high priority for ODY. Since the accuracy of the accelerometers was adequate to monitor aerobraking, the accelerometer design would not be enhanced for scientific studies. Nevertheless, ODY accelerometer measurements provided additional insight into the martian thermosphere. The scientific benefits of these data became generally accepted and MRO was the first mission to include the accelerometer as a facility experiment with a competition for selection of the facility team. With that status, the team could place measurement requirements on the project. This resulted in the significantly improved accuracy of MRO over ODY which resulted from modification of the ODY accelerometer electronics.

The potential exist to use accelerometer data to make additional contributions to our knowledge of the upper atmosphere. Higher spatial resolution could provide information on gravity wave dissipation processes, greater sensitivity would permit higher altitude measurements and could quantify the transition from the thermosphere to the exosphere, and using an orbit with a lower inclination would provide in situ longitudinal and diurnal structure. To assure success of future accelerometer experiments will require that the accelerometer be recognized as a scientific instrument and be given the same role in the spacecraft and mission design as is currently afforded to a camera or a spectrometer.

\section{Acknowledgment}

The authors would like to acknowledge NASA support through the Mars Data Analysis program and JPL support during the aerobraking operational phases of MGS, Odyssey and MRO.

\section{References}

${ }^{1}$ Lyons, D., “Aerobraking Magellan: Plan versus Reality,” Advances in the Astronautical Sciences, Vol. 87, Pt. 2, 1994, pp. 663-680.

2 Keating, G. M.; Hsu, N. C.; and Lyu, J., "Improved Thermospheric Model for the Venus International Reference Atmosphere,” Proceedings of 31st Scientific Assembly of COSPAR, Birmingham, England, 139, 1996.

${ }^{3}$ Croom, C. and Tolson, R., "Using Magellan Attitude Control Data to Study the Venusian Atmosphere and Various Spacecraft Properties,” AAS-94-141. AAS/AIAA Spaceflight Mechanics Meeting, Feb. 14-16, 1994.

4 Espiritu, R. and Tolson, R., "Determining Venusian Upper Atmosphere Characteristics Using Magellan Attitude Control Data,” AIAA/AAS Spaceflight Mechanics Meeting, Alburquerque, NM, February 13-16, 1995.

5 Tolson, R., et al., “Application of Accelerometer Data to Mars Global Surveyor Aerobraking Operations,” Journal of Spacecraft and Rockets, Vol 36, No 3, pp. 323-329, 1999.

6 Tolson, R., Keating, G., Noll, S., Baird, D., and Shellenberg, T., "Utilization of Mars Global Surveyor Accelerometer Data for Atmospheric Modeling,” Astrodynamics 1999, Vol 103, Advances in the Astronomical Sciences, 2000, pp. 1329-1346.

${ }^{7}$ Tolson, R., et al., “Application of Accelerometer Data to Mars Odyssey Aerobraking and Atmospheric Modeling,” Journal of Spacecraft and Rockets, Vol. 42, No. 3, pp. 435-443, 2005. 
${ }^{8}$ Graf, J., Zurek, R., Jones, J., Eisen, H., Johnson, M., Jai, B., “An Overview of the Mars Reconnaissance Orbiter Mission,” 2002 IEEE Aerospace Conference Proceedings, March 11-15, 2002

9 Johnston, M., Graf, J., Zurek, R., Eisen, H., Jai, B., “The Mars Reconnaissance Orbiter Mission

10 Justus, C., James, B., Bougher, S., Bridger, A., Haberle, R., Murphy, J. and Engel, S. "Mars-GRAM 2000: A Mars Atmospheric Model for Engineering Applications,” Advances in Space Research, vol. 29, p. 193-202, 2002

11 Justus, C., Duvall, A., Johnson, D., "Mars Global Reference Atmospheric Model (MARS-GRAM) and Database for Missions Design,” Mars Atmosphere Modeling and Observations Workshop, January 13 - 15, 2003, Granada, Spain.

12 Smith, J., Bell, J, “2001 Mars Odyssey Aerobraking,” AIAA 2002-4532, AIAA/AAS Astrodynamics Specialist Conference, Aug. 5-8, 2002.

13 Keating et al, “The Upper Atmosphere of Mars: First In Situ Measurements from an Orbiting Spacecraft,” Science, 279, 1672, 1998.

14 Dwyer, A., Tolson, R., Monk, M., Tartabini, P., “Development of a Monte Carlo Mars-GRAM Model for Mars 2001 Aerobraking Simulations,” Astrodynamics 2001, Vol. 109, Advances in Astronautics, pp. 1293-1308, 2002.

${ }^{15}$ Wilson, R. and Hamilton, K., “Comprehensive model simulation of thermal tides in the Martian atmosphere,” J. of Atm. Sciences, Vol. 53, No. 9, 1996 pp. 1290-1326.

${ }^{16}$ Forbes, J, et al, “Nonmigrating tides in the thermosphere of Mars,” J. Geophys. Res., 107(E11), 5113, 2002.

${ }^{17}$ Wilson, R. "Evidence for nonmigrating thermal tides in the Mars upper atmosphere from the Mars Global Surveyor Accelerometer Experiment”, Geophys. Res. Lett., 29 (7), 2002.

18 Baird, D., Tolson, R., Bougher, S., "Calculation of Zonal Winds Using Accelerometer and Rate Data from Mars Global Surveyor,” AIAA-2006-6390, Keystone, CO, August 2006.

19 Crowley, G. and Tolson, R., “Mars Thermospheric Winds from MGS and Odyssey Accelerometers,” AIAA-2006-6392, Keystone, CO, August 2006.

${ }^{20}$ Bougher, S., Bell, J., Murphy, J., Lopez-Valverde, M., and Wither, P., "Polar warming in the Mars thermosphere: Seasonal variations owing to changing insolation and dust distributions,” Geophysical Research Letters, Vol. 33, L02203, 2006. 